\title{
Resposta à Seleção para Características de Desempenho em um Rebanho de Seleção de Suínos ${ }^{1}$
}

\author{
Fabiano Veraldo da Costa Pita ${ }^{2}$, Lucia Galvão de Albuquerque ${ }^{3,4}$
}

\begin{abstract}
RESUMO - O objetivo do presente trabalho foi estimar respostas à seleção para as características de desempenho ganho de peso médio diário (GMD), idade (IF95) e número de dias de permanência no teste (ND95), necessários para atingir 95 kg de peso vivo, avaliadas nas raças Landrace (LD), Large White (LW) e Pietrain (PT), em um núcleo de seleção. Os valores genéticos dos animais foram preditos usando-se a metodologia de modelos mistos, aplicada a modelos animais. As estimativas de resposta foram determinadas através de regressão ponderada dos valores genéticos médios sobre o ano de nascimento dos animais, utilizando-se o método de Quadrados Mínimos Ponderado. As estimativas foram favoráveis para as três raças e ambos os sexos. Para o GMD estas foram, respectivamente para fêmeas e machos, de 9,0 e 9,5 g/dia/ano para a raça LD, 5,9 e 6,2 g/dia/ano para a raça $\mathrm{LW} \mathrm{e,} \mathrm{6,6} \mathrm{e} \mathrm{7,3} \mathrm{g/dia/ano} \mathrm{para} \mathrm{a} \mathrm{raça} \mathrm{PT.} \mathrm{Do} \mathrm{mesmo} \mathrm{modo,}$ para a IF95 estas foram de -0,52 e -0,55 dia/ano para LD e, - -0,98 e -1,14 dias/ano para PT, não havendo efeito linear significativo para esta característica na raça LW. Finalmente, para ND95 estas foram de -0,98 e -1,02 dias/ano para LD, - 0,74 e $-0,77$ dia/ano para LW e $-0,82$ e -0,88 dia/ano para PT. Estes resultados sugerem que o programa de seleção empregado no núcleo de seleção está sendo eficiente, com prováveis reflexos sobre o sistema total de produção (granjas multiplicadoras e comerciais).
\end{abstract}

Palavras-chave: ganho de peso médio diário, idade ao final do teste, número de dias no teste, tendência genética

\section{Selection Response for Performance Traits in a Swine Nucleus Herd}

\begin{abstract}
The aim of this work was to estimate selection response for performance traits average daily gain (ADG), off-test age (A95) and number of days on test (ND95), to reach $95 \mathrm{~kg}$ of live weight, evaluated for Landrace (LD), Large White (LW), and Pietrain (PT) breeds, in a nucleus herd. Breeding values were predicted using mixed model methodology. Estimates of response were determined using Weighted Least Squares method. The estimates were favourable for three breeds and both sex. For ADG these were, respectively for females and males, of 9.0 and $9.5 \mathrm{~g} /$ day/year for LD, 5.9 and $6.2 \mathrm{~g} /$ day/year for LW, and 6.6 and $7.3 \mathrm{~g} /$ day/year for PT. In the same way, for A95 these were of -0.52 and -0.55 day/year for LD, and -0.98 and -1.14 days/year for PT. Non significative linear effects were estimated for this trait at both sex of LW breed. Finally, for ND95 these were of -0.98 and -1.02 days/year for LD, -0.74 and -0.77 day/ year for LW, and -0.82 and -0.88 day/year for PT. These results suggest that the selection program applied at this nucleus is effective, and probably it is reflecting on whole production system.
\end{abstract}

Key Words: average daily gain, genetic trend, number of days on test, off-test age

\section{Introdução}

Nos núcleos de seleção de suínos, a característica de desempenho comumente utilizada para avaliar a capacidade de crescimento dos indivíduos é o ganho de peso médio diário (GMD), o qual, em geral, é mensurado em um período compreendido entre a desmama e o abate dos animais (fases de crescimento e terminação), sob condições de teste de granja.

Alternativamente ao GMD, a idade e o número de dias no teste, necessários para atingir um determinado peso padrão de abate, são também características que podem ser utilizadas para avaliação da taxa de crescimento de suínos. Ambas incorporam o conceito de unidade de mercado e vêm sendo consideradas, como critérios de seleção, principalmente nos países de suinocultura avançada. Segundo SULLIVAN e DEAN (1994), o programa nacional de avaliação genética de suínos do Canadá vem utilizando a idade para atingir $100 \mathrm{~kg}$ de peso vivo, como critério de seleção para desempenho, desde 1992.

Como o progresso genético para estas características de desempenho, em uma população de suínos, depende dos ganhos genéticos obtidos nos núcleos de seleção, torna-se determinante a avaliação periódica da eficiência do programa de melhoramento genético

\footnotetext{
1 Parte da dissertação de mestrado do primeiro autor (Curso de pós-graduação em Zootecnia, área de concentração em Genética e Melhoramento Animal, Faculdade de Ciências Agrárias e Veterinárias, UNESP/Campus de Jaboticabal), financiada pela FAPESP

2 Zootecnista. Endereço atual: Universidade Federal de Viçosa, curso de pós-graduação em Genética e Melhoramento. E.mail:fvcpita@bol.com.br

3 Professora assistente doutora. FCAV - UNESP/Jaboticabal. Depto. de Zootecnia, setor de Melhoramento Animal. E.mail: Igalb@fcav.unesp.br

4 Pesquisadora do CNPq.
} 
empregado nestes núcleos. Uma maneira de se avaliar essa eficiência é através da estimação da resposta à seleção (ou tendência genética) para as características de interesse.

Portanto, o objetivo do presente trabalho foi de estimar respostas à seleção para as características ganho de peso médio diário (GMD), idade (IF95) e número de dias no teste (ND95), necessários para atingir um peso vivo de $95 \mathrm{~kg}$, avaliadas em animais Landrace, Large White e Pietrain, submetidos a teste de granja em um núcleo de seleção.

\section{Material e Métodos}

Os dados de produção de suínos utilizados no presente trabalho pertencem à Granja Rezende S.A., que desenvolve atividades agropecuárias em Uberlândia (MG).

Foram utilizados registros de desempenho de 32.702 animais, sendo 17.836 da raça Landrace, 9334 da raça Large White e 5532 da raça Pietrain, nascidos entre 1991 e 1998. Este rebanho foi formado a partir da importação de animais de ambos os sexos da Inglaterra (animais Landrace e Large White) e da Alemanha (animais Pietrain). Após 1993, o rebanho foi fechado.

Os animais avaliados nasceram em instalações climatizadas, sendo o procedimento de equalização de leitegada realizado em dois momentos (cinco horas e quatro dias após o nascimento). A desmama ocorreu com 14 dias de idade. Após a saída da creche (em torno de 64 dias de idade), os animais de cada sexo foram alojados, separadamente, em galpões de crescimento e terminação. Nestes galpões o teste de granja foi iniciado com a pesagem dos animais após um período de adaptação de sete dias. Tanto para as fêmeas quanto para os machos a ração foi servida à vontade em baias coletivas. Os animais de ambos os sexos foram selecionados aos 145 dias de idade, com base em um índice que incluía as características GMD, espessura de toucinho e tamanho de leitegada.
Na Tabela 1 pode ser observada a estrutura dos dados analisados, após análises de consistência, para cada raça.

Os valores genéticos dos indivíduos, para as três características, foram preditos pela metodologia de modelos mistos (HENDERSON, 1949), aplicada a modelos animais, utilizando-se o programa computacional MTDFREML (BOLDMAN et al., 1993). Os modelos estatísticos utilizados para a predição, cuja porção aleatória foi determinada em análises prévias (PITA, 2000), foram:

Para as raças Large White e Pietrain: $y=X \beta+$

$$
\mathrm{Z}_{1} \mathrm{~g}+\mathrm{Wc}+\mathrm{e}
$$

Para a raça Landrace: $y=X \beta+Z_{1} g+Z_{2} m+W c+e$; em que: y é o vetor das observações; $\beta$, o vetor dos efeitos fixos; g, o vetor dos efeitos genéticos aditivos diretos dos animais; $\mu$, o vetor dos efeitos genéticos maternos dos animais; c, o vetor dos efeitos permanentes e comuns a membros da mesma leitegada; e, o vetor dos efeitos residuais; e $\mathrm{X}, \mathrm{Z}_{1}, \mathrm{Z}_{2} \mathrm{eW}$, as matrizes de incidência que associam os efeitos apropriados a y.

As pressuposições para os modelos foram:

$$
\begin{gathered}
\mathrm{E}[\mathrm{y}]=\mathrm{X} \beta, \mathrm{E}[\mathrm{g}]=0, \mathrm{E}[\mathrm{m}]=0, \mathrm{E}[\mathrm{c}]=0 \text { e } \mathrm{E}[\mathrm{e}]=0 \\
\operatorname{Var}[\mathrm{g}]=\mathrm{A} \sigma_{\mathrm{g}}^{2}, \operatorname{Var}[\mathrm{m}]=\mathrm{A} \sigma^{2}{ }_{\mathrm{m}}, \operatorname{Var}[\mathrm{c}]=\mathrm{I}_{\mathrm{NL}} \sigma^{2}{ }_{\mathrm{c}}, \\
\operatorname{Var}[\mathrm{e}]=\mathrm{I}_{\mathrm{N}} \sigma^{2}{ }_{\mathrm{e}} \\
\operatorname{Cov}[\mathrm{g}, \mathrm{m}]=0
\end{gathered}
$$

em que: $-\mathrm{NL}=$ número de leitegadas; $-\sigma_{\mathrm{g}}{ }^{2}=$ variância genética aditiva direta; $-\mathrm{N}=$ número de registros $-\sigma_{\mathrm{m}}{ }^{2}=$ variância genética materna; $-\mathrm{A}=$ numerador da matriz de parentesco; $-\sigma_{c}{ }^{2}=$ variância de ambiente permanente e comum (variância de leitegada); $-\mathrm{I}=$ matriz identidade; $-\sigma_{\mathrm{e}}^{2}=$ variância residual.

As covariâncias entre os efeitos genéticos e ambientais (permanentes e temporários), bem como entre os efeitos ambientais de diferentes animais, foram assumidas como zero.

$O$ vetor de efeitos fixos $(\beta)$ considerou como variável classificatória o grupo contemporâneo (GC),

\begin{tabular}{|c|c|c|c|c|c|c|}
\hline $\begin{array}{l}\text { Raça } \\
\text { Breed }\end{array}$ & $\begin{array}{l}\mathrm{N}^{\mathrm{o}} \text { de animais } \\
\text { N. of animals }\end{array}$ & $\begin{array}{l}\mathrm{N}^{\mathrm{o}} \text { de animais na } \mathrm{A}^{1} \\
N \text {. of animals in the } A^{1}\end{array}$ & $\begin{array}{l}\mathrm{N}^{\mathrm{o}} \text { de } \mathrm{GC} \mathrm{c}^{2} \mathrm{~s}^{2} \\
\text { N. of } C G{ }^{\prime} s^{2}\end{array}$ & $\begin{array}{c}\mathrm{N}^{\mathrm{O}} \text { de leitegadas } \\
\text { N. of litters }\end{array}$ & $\begin{array}{l}\mathrm{N}^{\circ} \text { de pais } \\
N \text {. of sires }\end{array}$ & $\begin{array}{c}\mathrm{N}^{\mathrm{o}} \text { de mães } \\
N . \text { of dams }\end{array}$ \\
\hline Landrace & 17.563 & 17.905 & 305 & 2708 & 154 & 1322 \\
\hline Large White & 8679 & 8894 & 213 & 1342 & 106 & 656 \\
\hline Pietrain & 5144 & 5317 & 190 & 1012 & 82 & 529 \\
\hline
\end{tabular}
além da idade e o peso, ambos ao início do teste, como covariáveis, respectivamente, para análise do GMD

Tabela 1- Estrutura dos dados analisados para cada raça Table 1 - Analyzed data structure for each breed

1 A - matriz de parentesco ( $A$ - relationship matrix).

2 GC - grupo contemporâneo (CG - contemporary group).

Rev. bras. zootec., 30(6S):2009-2016, 2001 
e do ND95. O GC foi definido como animais de mesmo sexo, mês e ano de nascimento e mesmo local de nascimento e teste.

As estimativas de resposta à seleção para as características GMD, IF95 e ND95, em ambos os sexos, foram obtidas por intermédio de análise de regressão pelo método de Quadrados Mínimos Ponderado. As variáveis dependentes foram o valor genético aditivo direto e materno médios (este último apenas para a raça Landrace) das características em cada ano de nascimento, enquanto a variável independente foi o ano de nascimento dos indivíduos. $O$ fator de ponderação foi o número de indivíduos avaliados por ano de nascimento.

\section{Resultados e Discussão}

Para a raça Landrace, as estimativas de herdabilidade do efeito direto e materno, utilizadas para predizer os valores genéticos, foram de, respectivamente, $0,28( \pm 0,03)$ e $0,02( \pm 0,01)$ para GMD,
$0,24( \pm 0,03)$ e $0,03( \pm 0,01)$ para IF95 e $0,24( \pm 0,03)$ e $0,01( \pm 0,01)$ para ND95. Para as raças Large White e Pietrain, as estimativas de herdabilidade do efeito direto foram de, respectivamente, $0,28( \pm 0,04)$ e 0,29 $( \pm 0,04)$ para GMD, $0,27( \pm 0,04)$ e $0,27( \pm 0,04)$ para IF95 e, 0,23 $( \pm 0,04)$ e $0,23( \pm 0,04)$ para ND95.

Na Tabela 2 podem ser observados por sexo, em cada raça, médias e desvios-padrão para as características analisadas.

Como pode ser observado, em geral, os animais machos apresentaram desempenho superior às fêmeas, embora para as raças Large White e Pietrain o desempenho dos animais de ambos os sexos tenha sido bastante semelhante.

$\mathrm{Na}$ Tabela 3 podem ser observadas as estimativas anuais de resposta à seleção para as características GMD, IF95 e ND95, em ambos os sexos e para as três raças, bem como a significância do efeito linear.

Para a característica GMD, verificou-se uma resposta à seleção para efeito genético aditivo direto

Tabela 2 - Médias e desvios-padrão, por sexo, para ganho de peso médio diário (GMD), idade ao final do teste (IF95) e número de dias de permanência no teste (ND95), para cada raça

Table 2 - Averages and standard deviations, by sex, for average daily gain (ADG), off-test age (A95) and number of days on test (ND95), for each breed

\begin{tabular}{lcccc}
\hline \multicolumn{5}{c}{ Características } \\
Traits \\
Raça & \multicolumn{5}{c}{ Sexo ${ }^{1}$} & GMD (kg/dia) & IF95 (dias) & ND95 (dias) \\
Breed & Sex $^{1}$ & ADG (kg/day) & A95 (days) & ND95 (days) \\
\hline Landrace & $\mathrm{M}$ & $0,886 \pm 0,109$ & $149,62 \pm 14,34$ & $80,54 \pm 13,71$ \\
& $\mathrm{~F}$ & $0,848 \pm 0,094$ & $152,31 \pm 12,43$ & $83,41 \pm 12,50$ \\
Large White & $\mathrm{M}$ & $0,912 \pm 0,126$ & $149,51 \pm 14,98$ & $78,85 \pm 14,63$ \\
& $\mathrm{~F}$ & $0,909 \pm 0,107$ & $149,01 \pm 12,65$ & $78,14 \pm 13,05$ \\
Pietrain & $\mathrm{M}$ & $0,716 \pm 0,093$ & $173,94 \pm 17,27$ & $103,35 \pm 17,49$ \\
& $\mathrm{~F}$ & $0,710 \pm 0,085$ & $174,47 \pm 15,82$ & $103,61 \pm 16,54$ \\
\hline
\end{tabular}

${ }^{1} \mathrm{M}$ - macho, F- fêmea ( $M$ - male, $F$ - female).

Tabela 3 - Estimativas anuais de resposta à seleção para as características ganho de peso médio diário (GMD), idade ao final do teste (IF95) e número de dias de permanência no teste (ND95), para as três raças e ambos os sexos

Table 3 - Annual selection response estimates for average daily gain (ADG), off-test age (A95) and number of days on test (ND95), for each breed and both sex

\begin{tabular}{|c|c|c|c|c|c|c|c|}
\hline \multirow{3}{*}{$\begin{array}{l}\text { Raça } \\
\text { Breed }\end{array}$} & \multicolumn{4}{|c|}{$\begin{array}{c}\text { Efeito genético aditivo direto } \\
\text { Direct additive genetic effect }\end{array}$} & \multicolumn{3}{|c|}{$\begin{array}{l}\text { Efeito genético materno } \\
\text { Maternal genetic effect }\end{array}$} \\
\hline & Sexo $^{1}$ & $\operatorname{GMD}(\mathrm{kg} / \mathrm{dia})$ & IF95 (dias) & ND95 (dias) & $\operatorname{GMD}(\mathrm{kg} / \mathrm{dia})$ & IF95 (dias) & ND95 (dias) \\
\hline & $\operatorname{Sex}^{1}$ & $A D G(\mathrm{~kg} /$ day $)$ & A95 (days) & ND95 (days) & $A D G(\mathrm{~kg} /$ day $)$ & A95 (days) & ND95 (days) \\
\hline \multirow[t]{2}{*}{ Landrace } & M & $0,0095 * *$ & $-0,55^{* *}$ & $-1,02 * *$ & $0,0006^{\mathrm{NS}}$ & $-0,05^{\mathrm{NS}}$ & $-0,04 * *$ \\
\hline & $\mathrm{F}$ & $0,0090 * *$ & $-0,52 * *$ & $-0,98 * *$ & $0,0006^{* *}$ & $-0,05^{\mathrm{NS}}$ & $-0,05 * *$ \\
\hline \multirow[t]{2}{*}{ Large White } & M & $0,0062 * *$ & $-0,35^{\mathrm{NS}}$ & $-0,77 * *$ & - & - & - \\
\hline & $\mathrm{F}$ & $0,0059 * *$ & $-0,32^{\mathrm{NS}}$ & $-0,74 * *$ & - & - & - \\
\hline \multirow[t]{2}{*}{ Pietrain } & M & $0,0073 * *$ & $-1,14 * *$ & $-0,88 * *$ & - & - & - \\
\hline & $\mathrm{F}$ & $0,0066^{* *}$ & $-0,98 * *$ & $-0,82 * *$ & - & - & - \\
\hline
\end{tabular}

$1 \mathrm{M}$ - macho, F- fêmea ( $M$ - male, $F$ - female).

${ }^{* *}$ efeito linear significativo a $1 \%$, NS - efeito linear não-significativo ( ${ }^{* *}$ significative linear effect at $1 \%, N S-$ non-significative linear effect).

Rev. bras. zootec., 30(6S):2009-2016, 2001 
favorável para as três raças, sendo de maior magnitude nos machos, entretanto, para a raça Large White a diferença entre os sexos não foi tão pronunciada como na Landrace e Pietrain.

Também para as características IF95 e ND95 a resposta à seleção foi em um sentido favorável, a qual foi um pouco maior para os machos.

Conforme pode ser observado, não houve efeito linear significativo ao longo dos anos apenas para IF95 na raça Large White, em ambos os sexos.

Com relação ao efeito genético materno, avaliado na raça Landrace, praticamente não houve resposta à seleção para este efeito nas três características avaliadas. Esta ausência de resposta pode estar relacionada com a pequena magnitude deste efeito.

Nas Figuras 1 e 2 podem ser observadas, respectivamente para os animais Landrace machos e fêmeas, as linhas de tendência genética para o efeito genético aditivo direto entre os anos de 1991 a 1998 , para as três características de desempenho. Nas Figuras 3 e 4 estas podem ser observadas para os animais Large White machos e fêmeas, respectivamente, e, nas Figuras 5 e 6 estas são apresentadas para os animais Pietrain machos e fêmeas, respectivamente, no período de 1993 a 1998.

Nas Figuras 3 e 4 pode-se observar que o comportamento não linear da resposta à seleção para IF95 na raça Large White foi semelhante nos dois sexos, verificando-se uma redução bastante grande da idade para atingir $95 \mathrm{~kg}$ de peso vivo entre os anos de 1994 e 1996 e, um pequeno aumento desta até 1998. Como o ND95, que apresentou resposta linear ao longo dos anos, está mais relacionado com o desempenho dos animais durante o período de teste e a IF95 abrange, além do período de teste, o período pré-teste, talvez esta redução pronunciada da IF95 entre estes anos possa ser devida ao efeito de algum fator (decisão) anterior à entrada no teste dos indivíduos desta raça.

As estimativas de resposta à seleção observadas para GMD nos animais Landrace foram superiores à estimativa de 2,4 gramas/dia/ano obtida para esta mesma raça por KOVAC e GROENEVELD (1990). Para os animais Large White as estimativas obtidas foram superiores à de 1,0 grama/dia/ano obtida por PANETO (1998) ao analisar um rebanho elite do noroeste do Estado de São Paulo. Em relação aos animais Pietrain, KOVAC e GROENEVELD (1990) verificaram estimativa de 6,1 gramas/dia/ano, sendo esta semelhante às estimativas observadas neste estudo.
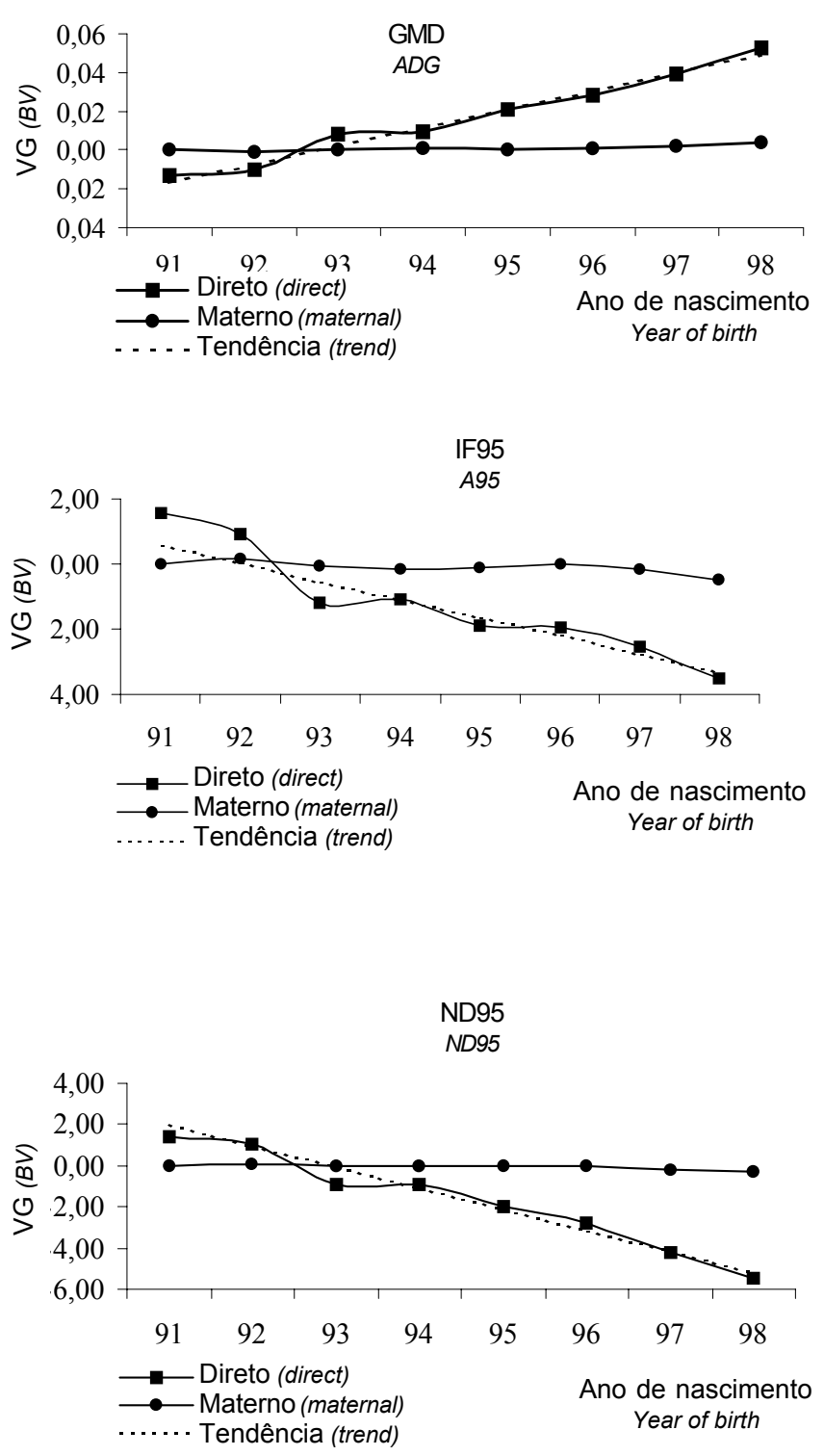

Figura 1- Valores genéticos médios (VG) dos animais Landrace machos, para as características ganho de peso médio diário (GMD), idade (IF95) e número de dias (ND95) para atingir $95 \mathrm{~kg}$ de peso vivo.

Figure 1 - Average breeding values (BV) of male Landrace, for average daily gain (ADG), off-test age (A95) and number of days on test (ND95).

Para a característica IF95, as estimativas obtidas para os animais Landrace e Large White foram inferiores às estimativas de - $0,82 \mathrm{e}-0,54 \mathrm{dia} / \mathrm{ano}$, respectivamente, observadas por HUDSON e KENNEDY (1985) e, menores ainda que as obtidas por SULLIVAN e DEAN (1994), que verificaram estimativas de $-1,3$ dias/ano e $-0,9$ dia/ano, 

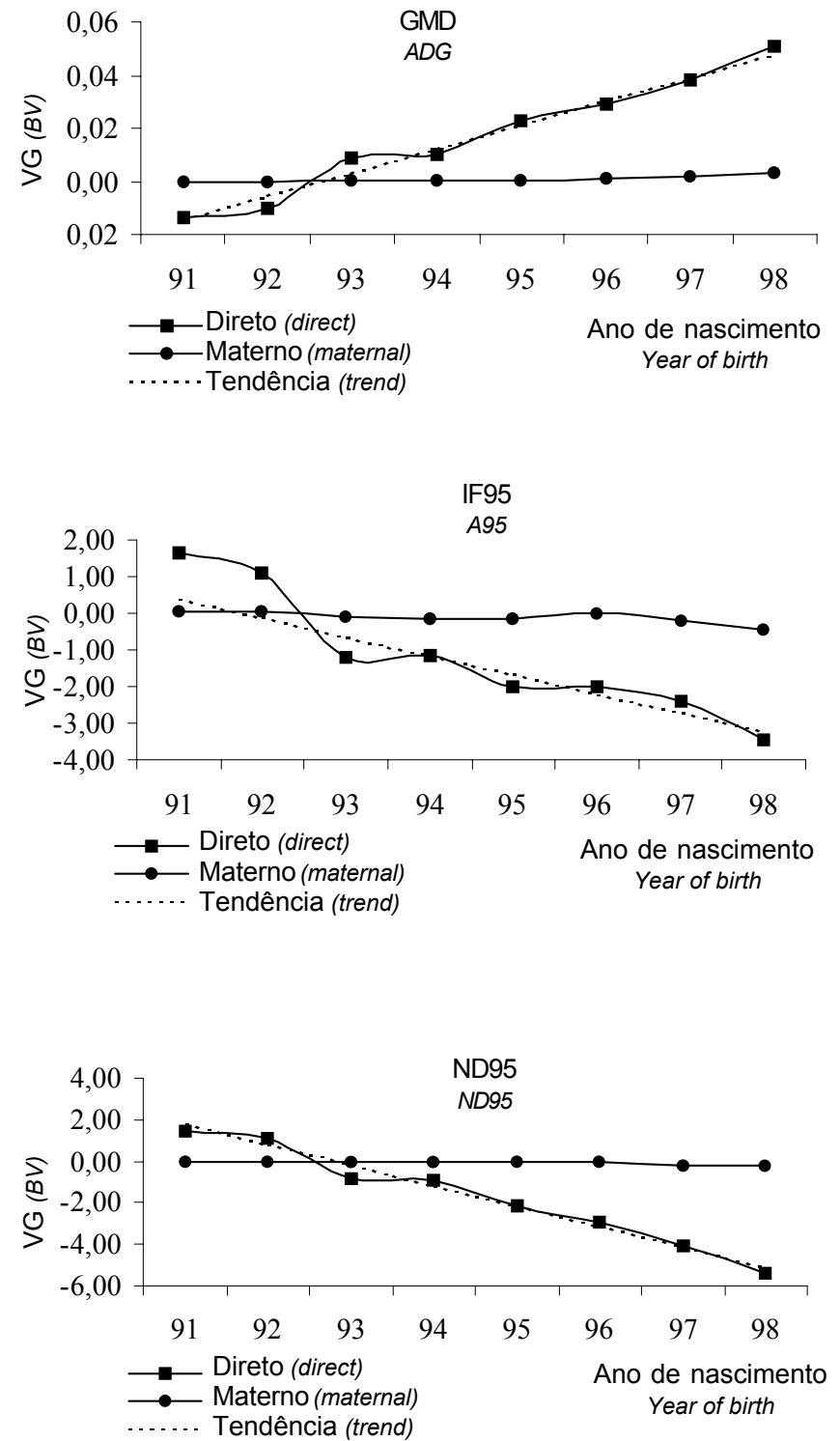

Figura 2 - Valores genéticos médios (VG) dos animais Landrace fêmeas, para as características ganho de peso médio diário (GMD), idade (IF95) e número de dias (ND95) para atingir $95 \mathrm{~kg}$ de peso vivo.

Figure 2 - Average breeding values (BV) of female Landrace, for average daily gain (ADG),off-test age (A95) and number of days on test (ND95).
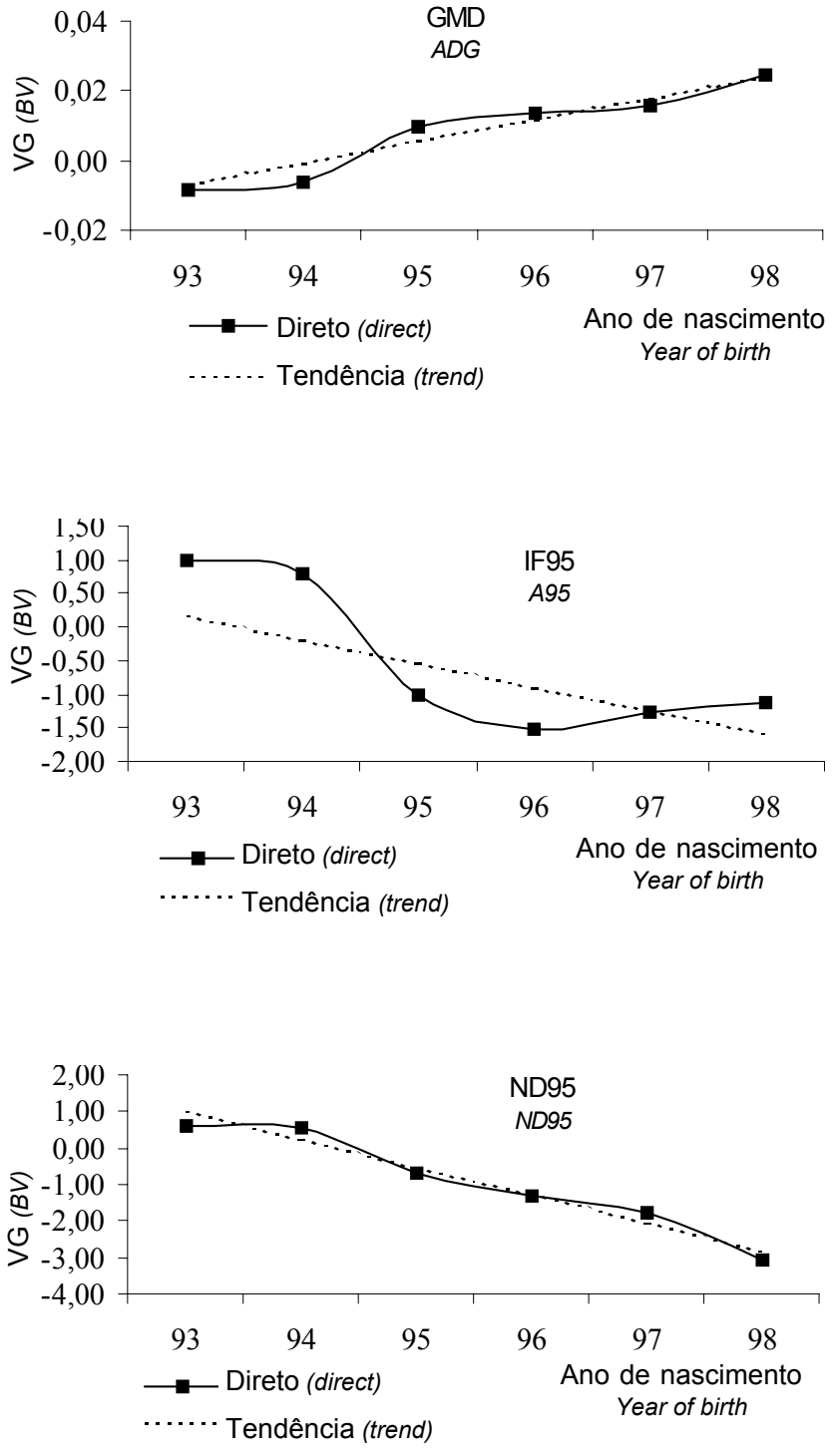

Figura 3 - Valores genéticos médios (VG) dos animais Large White machos, para as características ganho de peso médio diário (GMD), idade (IF95) e número de dias (ND95) para atingir $95 \mathrm{~kg}$ de peso vivo.

Figure 3 - Average breeding values (BV) of male Large White, for average daily gain (ADG), off-test age (A95) and number of days on test (ND95). 

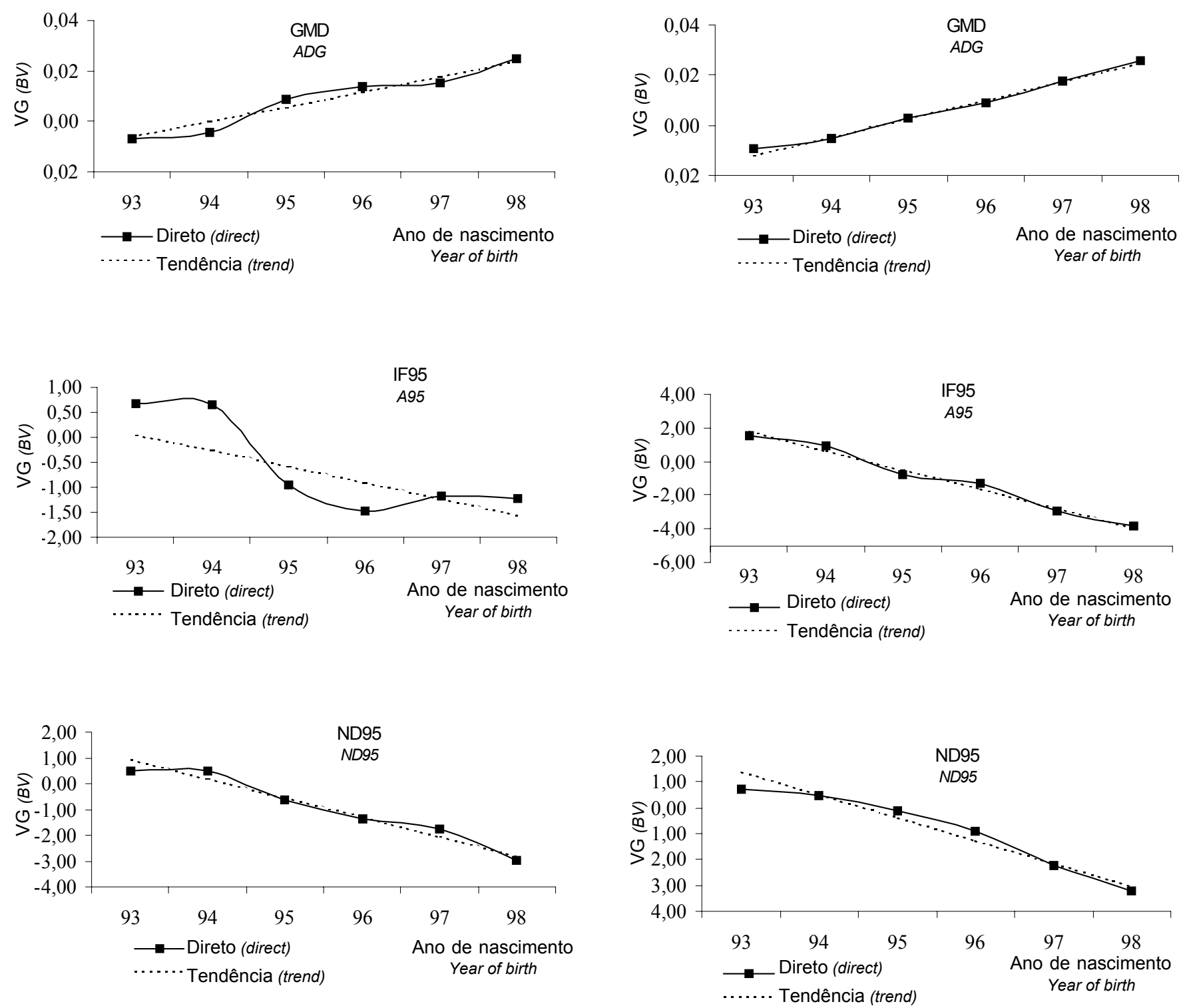

Figura 4 - Valores genéticos médios (VG) dos animais Large White fêmeas, para as características ganho de peso médio diário (GMD), idade (IF95) e número de dias (ND95) para atingir $95 \mathrm{~kg}$ de peso vivo.

Figure 4 - Average breeding values (BV) of female Large White, for average daily gain (ADG), off-test age (A95) and number of days on test (ND95).

Figura 5 - Valores genéticos médios (VG) dos animais Pietrain machos, para as características ganho de peso médio diário (GMD), idade (IF95) e número de dias (ND95) para atingir $95 \mathrm{~kg}$ de peso vivo.

Figure 5 - Average breeding values (BV) of male Pietrain, for average daily gain (ADG), off-test age (A95) and number of days on test (ND95).

Rev. bras. zootec., 30(6S):2009-2016, 2001 
Tabela 4 - Estimativas de resposta à seleção acumulada após um período de 10 anos, e expressas como porcentagem da média fenotípica observada no período, para ganho de peso médio diário (GMD), idade ao final do teste (IF95) e número de dias de permanência no teste (ND95), para cada raça

Table 4 - Estimates of selection response accumulated after 10 years, and as percentage of phenotypic average observed in the period, for average daily gain (ADG), off-test age (A95), and number of days on test (ND95), for each breed

\begin{tabular}{|c|c|c|c|c|c|}
\hline \multirow{3}{*}{$\begin{array}{l}\text { Raça } \\
\text { Breed }\end{array}$} & \multirow{3}{*}{$\begin{array}{l}\text { Resposta à seleção } \\
\text { Selection response }\end{array}$} & \multirow{3}{*}{$\begin{array}{l}\text { Sexo }^{1} \\
\operatorname{Sex}\end{array}$} & \multicolumn{3}{|c|}{$\begin{array}{c}\text { Características } \\
\text { Traits } \\
\end{array}$} \\
\hline & & & GMD (kg/dia) & IF95 (dias) & ND95 (dias) \\
\hline & & & $A D G(\mathrm{~kg} /$ day $)$ & A95 (days) & ND95 (days) \\
\hline \multirow[t]{4}{*}{ Landrace } & acumulada (10 anos) & $\mathrm{M}$ & 0,095 & $-5,5$ & $-10,2$ \\
\hline & accumulated (10 years) & $\mathrm{F}$ & 0,090 & $-5,2$ & $-9,8$ \\
\hline & \% média fenotípica & M & 1,07 & $-0,37$ & $-1,26$ \\
\hline & $\%$ of phenotypic average & $\mathrm{F}$ & 1,06 & $-0,34$ & $-1,17$ \\
\hline \multirow[t]{4}{*}{ Large White } & acumulada (10 anos) & M & 0,062 & - & $-7,7$ \\
\hline & accumulated ( 10 years) & $\mathrm{F}$ & 0,059 & - & $-7,4$ \\
\hline & \% média fenotípica & $\mathrm{M}$ & 0,68 & - & $-0,98$ \\
\hline & $\%$ of phenotypic average & $\mathrm{F}$ & 0,65 & - & $-0,95$ \\
\hline \multirow[t]{4}{*}{ Pietrain } & acumulada (10 anos) & M & 0,073 & $-11,4$ & $-8,8$ \\
\hline & accumulated (10 years) & $\mathrm{F}$ & 0,066 & $-9,8$ & $-8,2$ \\
\hline & \% média fenotípica & M & 1,02 & $-0,66$ & $-0,85$ \\
\hline & $\%$ of phenotypic average & $\mathrm{F}$ & 0,93 & $-0,56$ & $-0,79$ \\
\hline
\end{tabular}

${ }^{1} \mathrm{M}$ - macho, F- fêmea (M- male, F-female).
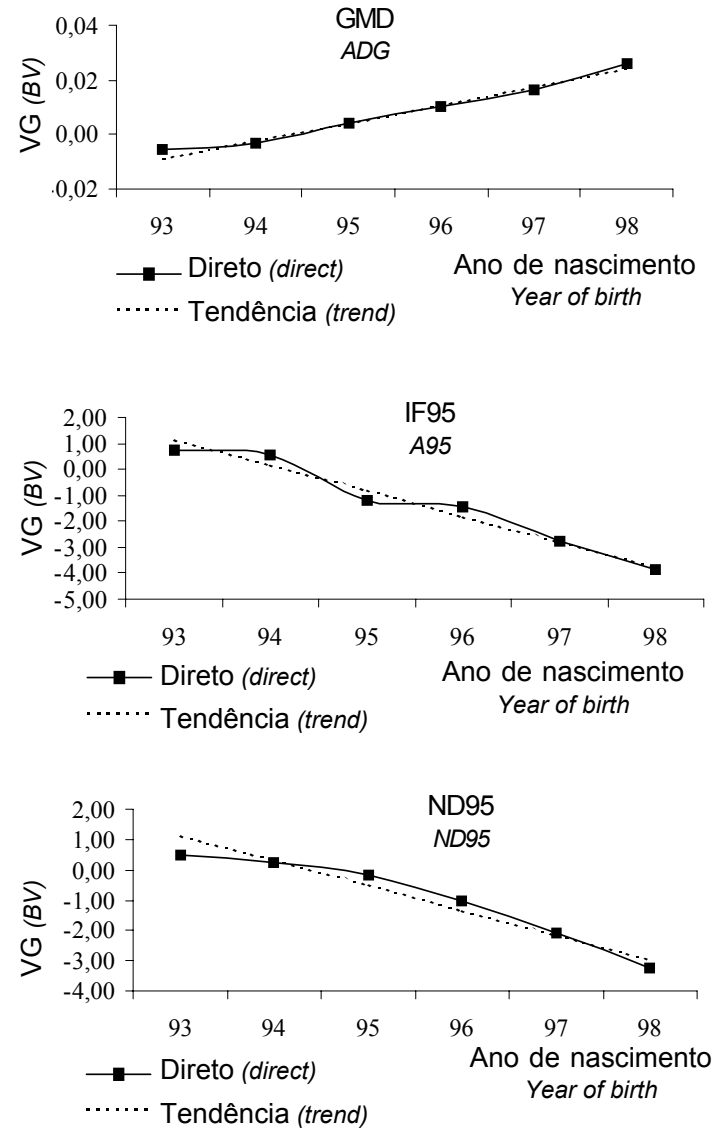

Figura 6 - Valores genéticos médios (VG) dos animais Pietrain fêmeas, para as características ganho de peso médio diário (GMD), idade (IF95) e número de dias (ND95) para atingir $95 \mathrm{~kg}$ de peso vivo.

Figure 6 - Average breeding values (BV) of female Pietrain, for average daily gain (ADG), off-test age (A95) and number of days on test (ND95). respectivamente, para estas duas raças, no Canadá. Na literatura consultada não foram encontradas estimativas de resposta à seleção para a característica ND95.

Conforme pode ser observado na Tabela 4, considerando-se as estimativas de resposta anual observadas no período estudado, haveria, após um período de 10 anos, redução em torno de 5,5 dias na IF95 para os animais Landrace e em torno de 10 a 11 dias para os animais Pietrain, o que é bastante significativo, considerando-se os custos diários de um suíno (alimentação, limpeza, tempo de ocupação de instalações etc). Já a resposta à seleção, expressa como porcentagem da média fenotípica observada no período estudado, sugere ganhos genéticos favoráveis e próximos de $1 \%$ ao ano para as características GMD e ND95, sendo que para IF95 esta porcentagem foi bem menor (principalmente para a raça Landrace).

\section{Conclusões}

Os resultados sugerem que o programa de melhoramento atualmente em prática permitiu a obtenção de progresso genético no núcleo de seleção, em relação ao efeito genético aditivo direto, para as três características de desempenho analisadas (GMD, IF95 e ND95), nas três raças e em ambos os sexos. Conseqüentemente, espera-se também um ganho genético em todo o sistema de produção (granjas multiplicadoras e comerciais). 


\section{Agradecimento}

À FAPESP (Fundação de Amparo à Pesquisa do Estado de São Paulo), pelo financiamento desta pesquisa, e à Granja Rezende S.A., pelo fornecimento dos dados utilizados neste estudo.

\section{Referências Bibliográficas}

BOLDMAN, K.G., KRIESE, L.A., VAN VLECK, L.D. et al. 1993. A manual for use of MTDFREML. Lincoln: USDAARS. $120 \mathrm{p}$.

HENDERSON, C.R. 1949. Estimation of changes in herd environment. J. Dairy Sci., 32:706-711.

HUDSON, G.F.S., KENNEDY, B.W. 1985. Genetic trend of growth rate and backfat thickness of swine in Ontario. J. Anim. Sci., 61(1):92-97.

KOVAC, M., GROENEVELD, E. 1990. Genetic and environmental trends in German swine herdbook populations. J. Anim. Sci., 68(11):3523-3535.

PANETO, J.C.C. Comparações entre as tendências genéticas e econômicas de um rebanho elite de suinos. Jaboticabal, SP: FCAV/UNESP, 1998. 90p. Dissertação (Mestrado em Zootecnia) - Faculdade de Ciências Agrárias e Veterinárias/ Universidade Estadual Paulista, 1998.
PITA, F.V.C. Modelos para avaliação genética e comparação de características de desempenho para a seleção de suínos. Jaboticabal, SP, FCAV/UNESP, 2000. 157p. Dissertação (Mestrado em Zootecnia) - Faculdade de Ciências Agrárias e Veterinárias/Universidade Estadual Paulista, 2000.

SULLIVAN, B.P., DEAN, R. National genetic evaluations for swine in Canada. In: WORLD CONGRESS ON GENETICS APPLIED TO LIVESTOCK PRODUCTION, 5, 1994, Guelph. Proceedings...Guelph, 1994. p.382-385.

Recebido em: 07/02/01

Aceito em: 23/05/01 